\title{
An endogenous F-box protein regulates ARGONAUTE1 in Arabidopsis thaliana
}

\author{
K Earley, MR Smith, R Weber, BD Gregory and RS Poethig*
}

\begin{abstract}
ARGONAUTE1 (AGO1) mediates microRNA- and small interfering RNA-directed posttranscriptional gene silencing in Arabidopsis thaliana. Mutant alleles of SQUINT (SQN) slightly reduce AGO1 activity and have weak effects on shoot morphology. A screen for mutations that suppress the sqn phenotype produced loss-of-function mutations in the Fbox gene FBW2. Mutations in FBW2 not only suppress sqn but also suppress many of the developmental phenotypes of weak, but not null, alleles of AGO1 by increasing AGO1 protein levels. Conversely, over-expression of FBW2 decreases the abundance of the AGO1 protein but not AGO1 messenger RNA, further indicating that FBW2 regulates AGO1 protein levels. fbw2 mutants have no obvious morphological phenotype, but display a reduced sensitivity to abscisic acid (ABA) that can be attributed to increased AGO1 activity. Our results indicate that FBW2 is a novel negative regulator of $\mathrm{AGO} 1$ and suggest that it plays a role in $\mathrm{ABA}$ signalling and/or response.
\end{abstract}

\section{Background}

Argonaute proteins are core components of the RNAinduced silencing complex (RISC) [1-3]. These proteins use microRNAs (miRNAs) and/or small interfering RNAs (siRNAs) as guides to direct RISC to a specific site in target mRNAs, resulting in the cleavage or translational repression of these target mRNAs. Some Argonaute proteins also promote transcriptional repression through their effect on chromatin structure [2-4].

ARGONAUTE1 (AGO1) is one of 10 Argonaute proteins in Arabidopsis thaliana [2,5]. Genetic analyses [6-9], as well as the identification of the small RNAs that copurify with AGO1 [10,11], indicate that AGO1 plays a central role in both miRNA and siRNA-mediated RNA silencing. Arabidopsis is exquisitely sensitive to the level of AGO1 activity, as evident from the broad range of phenotypes displayed by hypomorphic mutations of this gene $[5,6,8,12]$. In wild-type plants, the expression of AGO1 is maintained at a constant level by a negative feedback loop involving miR168. AGO1 is a target of miR168 and negatively regulates its own activity by promoting the activity and stability of miR168 $[9,13]$ and by promoting the activity of siRNAs derived from the AGO1 transcript [14]. AGO1 activity is negatively regulated by $\mathrm{PNH} / \mathrm{ZLL} /$

* Correspondence: spoethig@sas.upenn.edu

1 Department of Biology, University of Pennsylvania, Philadelphia PA 19104 USA

Full list of author information is available at the end of the article
AGO10 [15] and positively regulated by SQUINT (SQN), the Arabidopsis orthologue of the protein chaperone, Cyclophilin-40 [12].

Null alleles of $S Q N$ have a morphological phenotype that is nearly identical to the phenotype of weak loss-offunction alleles of $A G O 1$ [12]. In order to identify genes involved in AGO1-mediated processes, we screened for mutations that suppress the phenotype of sqn-1. This screen yielded several alleles of the F-box gene $F B W 2$. Here we show FBW2 is a negative regulator of AGO1 and controls the sensitivity of plants to the hormone abscisic acid.

\section{Results}

\section{Mutations in FBW2 rescue the sqn phenotype}

Previously we found that SQN directly or indirectly promotes AGO1 activity [12]. In particular, we showed that the phenotype of loss-of-function alleles of $S Q N$ can be largely, if not entirely, explained by a reduction in the activity of AGO1.

In order to study the mechanism of this regulation, we screened for ethyl methanesulfate (EMS)-induced mutations that suppress the phenotype of the null allele, sqn-1. sqn-1 transiently delays leaf initiation, accelerates the juvenile-to-adult transition and produces aberrant spacing of flowers in the inflorescence and an increase in carpel number [16] (Figure 1A and 1B). Three allelic mutations that partially suppress all of these phenotypes 

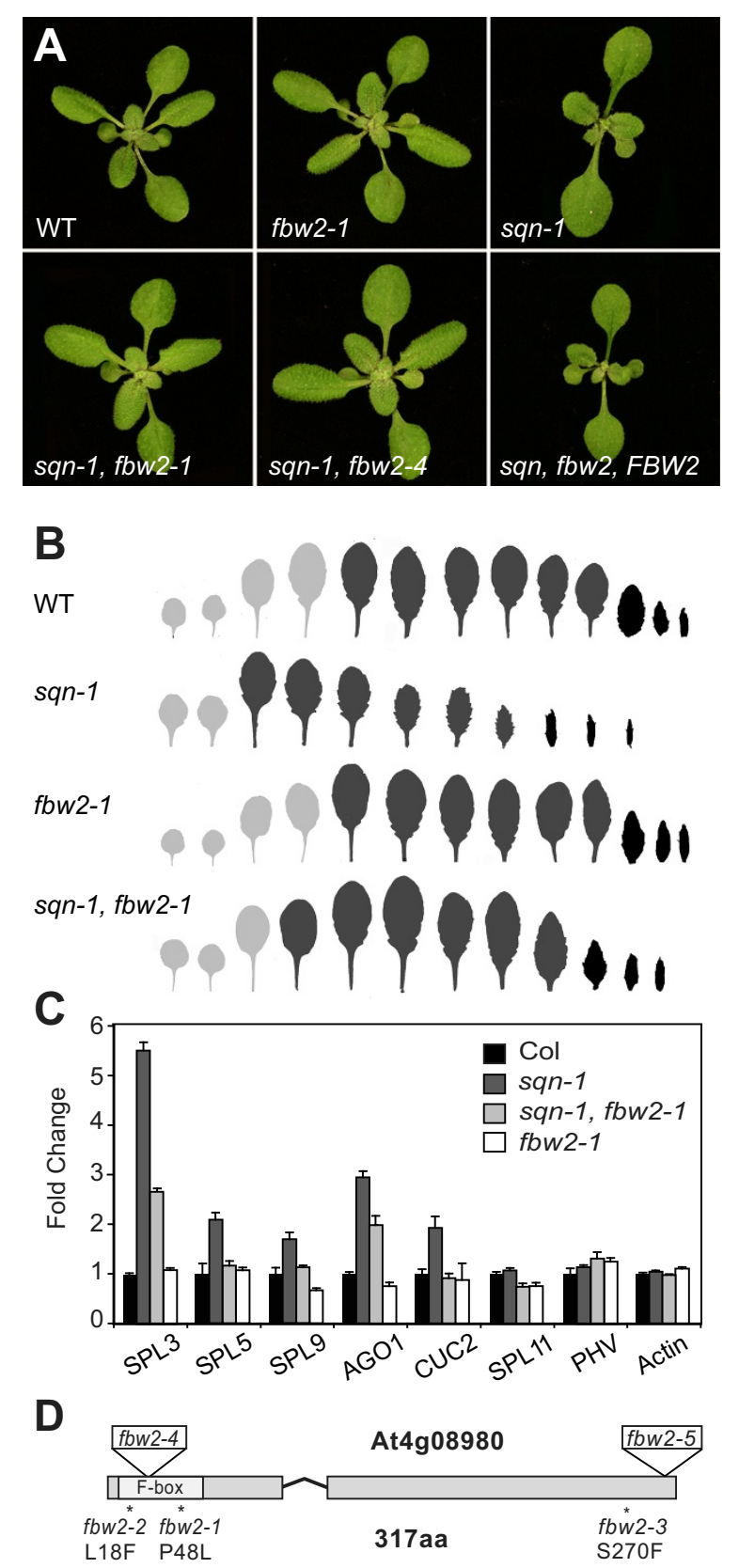

Figure 1 Loss-of-function mutations of FBW2 suppress the phenotype of sqn-1. (A) An 18-day-old rosettes of wild-type (WT), fbw2-1, sqn-1, sqn-1 fbw2-1, sqn-1 fbw2-4 and sqn-1 fbw2-1 plants containing a genomic pFBW2::FBW2-FLAG construct. Rosettes are same magnification. (B) Leaf shape of successive juvenile (light grey) adult (dark grey) and cauline leaves (black) in WT, fbw2-1, sqn-1 and san-1 fbw2-1 plants. Juvenile leaves were defined by the absence of abaxial trichomes $(n=$ $24 ; \pm$ standard deviation). (C) Relative abundance of miRNA targets in various genotypes as measured by quantitative-real time polymerase chain reaction. Target genes were normalized to EIF4. Actin was used as a non-target control ( \pm standard deviation). (D) The genomic structure of FBW 2 and the amino acid changes produced by fbw2-1, fbw2-2 and fbw2-3. The locations of the T-DNA insertions in fbw2-4 (SALK_144548) and fbw2-5 (SALK_071588) are also illustrated. were identified in this screen (Figure 1A, B and 1D). In addition to their effect on the morphological phenotype of $s q n-1$, these alleles decreased the expression of several miRNA-targeted genes (SPL3, SPL5, SPL9, AGO1, CUC2) previously shown to be over-expressed in sqn-1 [12] (Figure 1C). All three mutations had no obvious effect on shoot morphology or gene expression in the absence of sqn-1 (Figure 1A, B and 1C).

Using a map-based approach, we determined that this suppressor corresponds to FBW2 (F-BOX WITH WD-40 2) [17]. All three alleles change conserved residues in the predicted FBW2 protein (Figure 1D). Furthermore, a $\sim 4,700$-bp genomic construct expressing FBW2 under its native promoter ( $p F B W 2:: F B W 2-F L A G)$ restored the original $s q n-1$ phenotype when transformed into $s q n-1$ fbw2-1 (Figure 1A). Two additional mutations of FBW2 were identified in the SALK collection of T-DNA insertions [18], SALK_144548 (fbw2-4) and SALK_071588C (fbw2-5; Figure 1D). Like the point mutations recovered in our screen, both of these mutations had no obvious morphological phenotype but nearly completely suppressed the phenotype of $s q n-1$ in double mutants (Figure 1A). A real time polymerase chain reaction (RT-PCR) of the $F B W 2$ transcript in $s q n-1 f b w 2$ double mutants revealed that $f b w 2-2$ and $f b w 2-3$ have no effect on the abundance of this transcript, $f b w 2-1$ and $f b w 2-5$ reduce, but do not eliminate, the transcript, and that $f b w 2-4$ has no detectable FBW2 mRNA (Additional File 1: Figure S1A). We conclude that loss-of-function mutations of $F B W 2$ suppress the phenotype of $s q n-1$.

$F B W 2$ is predicted to encode a 317 -amino acid protein with an N-terminal F-box domain [17] (Figure 1D). Although FBW2 was originally described as having a WD-40 domain [17], we found no evidence for the presence of a canonical WD-40 domain in this protein. A number of other predicted proteins in the Arabidopsis genome have sequence similarity to FBW2, but the similarity between these proteins is quite low (less than $32 \%$ identical), which suggests that FBW2 may be functionally unique (Additional File 1: Figure S1B). FBW2 is highly conserved in flowering plants (Additional File 1: Figure $\mathrm{S1C}$ ), but is absent in the alga, Chlamydomonas reinhardtii. Interestingly, FBW2 has no strong sequence similarity to the Polerovirus P0 protein, an F-box protein that suppresses posttranscriptional gene silencing by destabilizing AGO1 $[19,20]$.

\section{Loss of FBW2 rescues hypomorphic ago mutations}

Previous work from our laboratory suggested that the sole function of SQN is to promote the activity of AGO1 [12]. Given that $f b w 2$ rescues null mutations of $S Q N$, we recognized that FBW2 cannot function through SQN. A reasonable alternative hypothesis is that $f b w 2$ mutations suppress $s q n-1$ by increasing the activity of AGO1. A pre- 
diction of this hypothesis is that $f b w 2$ mutations should be capable of suppressing at least some hypomorphic alleles of $A G O 1$ but should have no effect on the phenotype of a null allele. In order to test this hypothesis, we crossed $f b w 2-1$ and $f b w 2-4$ to 4 weak ago 1 mutant alleles (ago1-25, ago1-27, ago1-45, and ago1-46) and the null allele, ago 1-36 [8,12,21]. As predicted, $f b w 2-1$ and $f b w 2-4$ partially rescued the developmental and molecular phenotypes of all four hypomorphic ago1 mutations. Double mutants had a faster rate of leaf initiation, a later onset of abaxial trichomes, and larger and less serrated leaves than ago1 single mutant plants (Figure $2 \mathrm{~A}$ and $2 \mathrm{~B}$ ). They also displayed a decrease in the expression of many of the miRNA-targeted genes upregulated in ago1 single mutants $[9,12]$ (Figure 2D). In contrast, we observed no difference between the phenotype of ago1-36 single mutants and ago1-36 fbw2-1 double mutants (Figure 2C). These observations suggest that FBW2 either acts upstream of AGO1 or affects AGO1 activity.

The observed decrease in miRNA-regulated transcripts in sqn fbw2 and ago1 fbw2 compared with sqn and ago1 (Figure 1C and Figure 2D) could be explained by an increase in the accumulation of the miRNAs that target these transcripts for degradation. We did not favour this hypothesis because our genetic evidence suggests that FBW2 acts through AGO1, and ago1 hypomorphic alleles have limited effects on miRNA levels [9,12] (Figure 2E). Nevertheless, we compared the level of several miRNAs in $s q n f b w 2$ and ago1 fbw2 double mutants with $s q n$ and ago1 (Figure 2E). As predicted, sqn-1 and two hypomorphic ago1 alleles had weak or no effect miRNA levels and there was no significant difference between the miRNA levels in these single mutants and $s q n-1$ fbw 2 and ago1 $f b w 2$ double mutants (Figure 2E). Individually, fbw2 mutations also had no effect on miRNA levels (Figure $2 \mathrm{E})$. Thus, the reduction in the abundance of miRNA-regulated transcripts in $s q n f b w 2$ and ago1 fbw2 double mutants is not the result of an increase in miRNA expression.

\section{fbw2 mutations increase the abundance of AGO1}

We reasoned that if $f b w 2$ mutations suppress $s q n-1$ by increasing the activity of AGO1, it should be possible to replicate this effect by simply increasing the dose of AGO1. In order to test this hypothesis, we transformed a pAGO1::FLAG-AGO1 construct into sqn-1. This construct expresses a FLAG-AGO1 translational fusion under the regulation of the endogenous $A G O 1$ promoter. Numerous sqn-1 plants expressing this construct had near-WT phenotypes (Figure 3A). Indeed, the phenotypes of several of these sqn-1 pAGO1::FLAG-AGO1 lines were essentially identical to $s q n-1 f b w 2$ double mutants (Figure 3A). Western blots demonstrated that, as expected, sqn-1 pAGO1::FLAG-AGO1 plants had slightly more AGO1 protein than sqn-1 (Figure 3B). This result provides additional support for the conclusion that the phenotype of $s q n$ mutations is a consequence of a reduction in AGO1 activity--a conclusion that was originally based entirely on the phenotypic similarity between $s q n$ and ago 1 mutations and the genetic interaction between these mutations [12]. It also supports the hypothesis that fbw2 suppresses $s q n-1$ by increasing the activity of AGO1. As a direct test of this hypothesis, we compared AGO1 protein levels in wild-type, sqn-1, ago1-25, ago145 , and combinations of these mutations with $f b w 2$. We found that $s q n-1$ produced a small but reproducible decrease in AGO1 in both 14-day old leaf tissue and floral tissue, and that $f b w 2-1$ and $f b w 2-4$ suppressed this effect; $s q n-1$ fbw2 double mutants had approximately the same amount of AGO1 as wild-type plants (Figure 3B and Figure 4C). Similar results were obtained with hypomorphic alleles of AGO1: ago1-25 and ago1-45 had reduced amounts of AGO1 protein compared to wild-type plants, and $f b w 2-1$ and $f b w 2-4$ corrected this defect (Figure 3C). We conclude that $f b w 2$ suppresses the phenotype of $s q n$ 1, ago1-25 and ago1-45 by increasing the abundance of AGO1.

We also examined the genetic interaction between $f b w 2$ and several mutations that interfere with the biogenesis or stability of miRNAs, specifically $s e-1$ (Figure $5 \mathrm{~A}$ and 5B), hst-3 (Figure 5C and 5D), hyl1-2 (Figure 5E and 5F), and hen1-6 (Figure 5G and 5H). In every case, double mutants had stronger vegetative phenotypes than the single mutants. Although we were initially surprised by this result, we recognized that the phenotypes of these double mutants are remarkably similar to the phenotype of plants transformed with a miR168-resistant version of AGO1, which results in the over-expression of AGO1 (Figure 5K) $[9,13]$. In order to determine if this was a reasonable explanation for the effect of $f b w 2$, we introduced pAGO1::FLAG-AGO1 into se-1 and hst-3 (Figure 5I and $5 \mathrm{~J})$ and also assayed AGO1 proteins levels in single and double mutant plants (Figure 5L). Many hst-3 pAGO1::FLAG-AGO1 and se-1 pAGO1::FLAG-AGO1 primary transformants had phenotypes that were almost identical to $h s t-3 f b w 2$ and $s e-1 f b w 2$, and strongly resembled plants containing miR168-resistant AGO1 mRNA constructs (Figure $5 \mathrm{H}-\mathrm{J}$ ). Consistent with this observation, western blots revealed increased levels of AGO1 in se-1 fbw2, hen1-2 fbw2 and hst-3 fbw2 double mutants compared to the single mutants (Figure $5 \mathrm{~L}$ ). These observations support the conclusion that $f b w 2$ enhances the phenotypes of se-1, hst-3, hyl1-2 and hen 1- 6 by increasing AGO1 protein levels.

\section{Over-expression of FBW2 phenocopies ago 1 mutants}

F-box proteins are part of the E3 ubiquitin ligase complex, a protein complex that targets substrates for ubiq- 

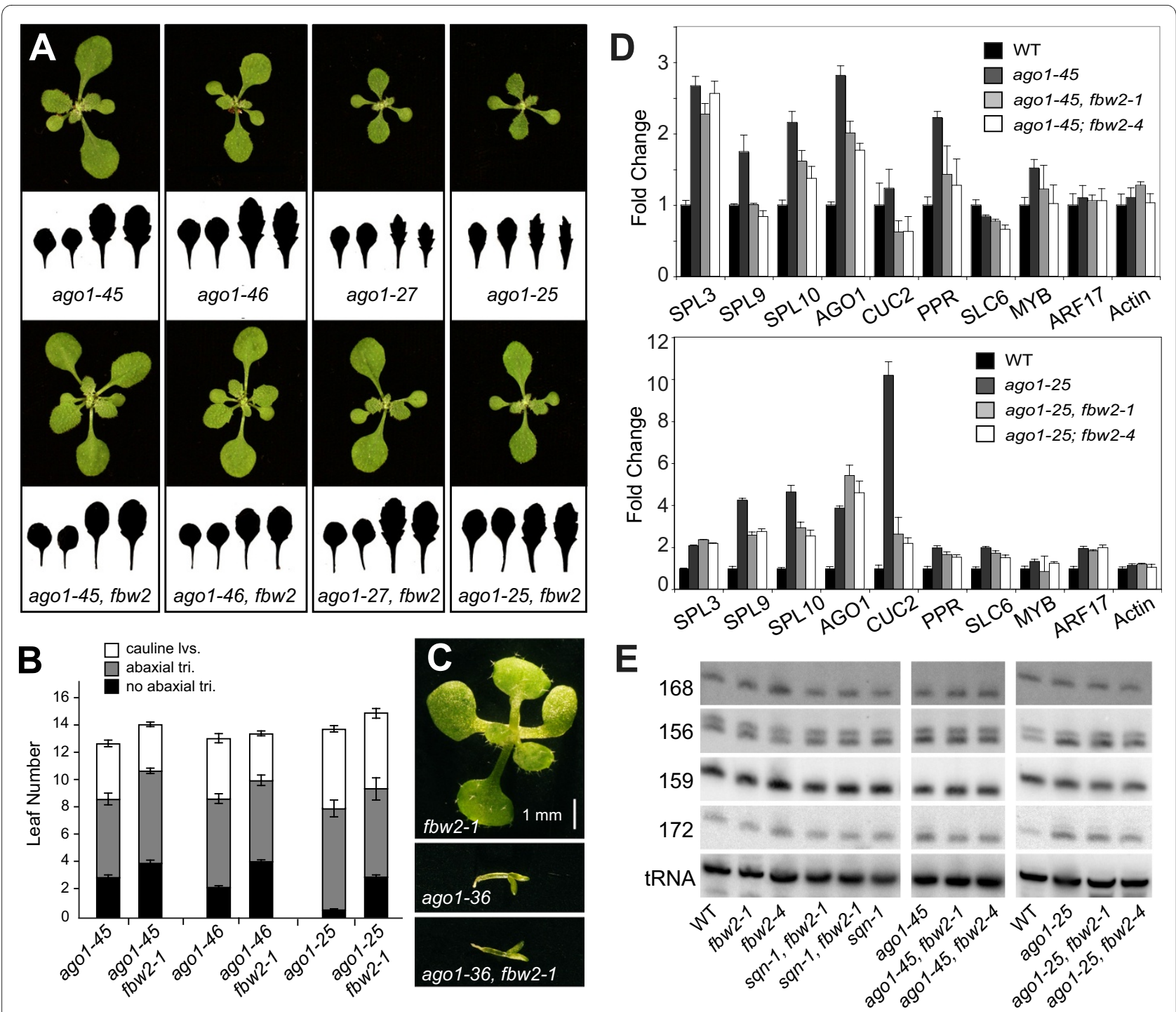

Figure 2 Mutations in FBW2 suppress hypomorphic ago 1 mutations. (A) Eighteen-day-old rosettes of ago 1 mutants and ago 1 fbw2-1 double mutants. The shapes of leaves 1-4 are also illustrated. (B) The number of juvenile, adult and cauline leaves in ago 1 and ago 1 fbw2-1 double mutants ( \pm standard deviation). (C) 14-day-old fbw2-1, ago 1-36 and ago 1-36 fbw2-1 double mutants grown on MS media. (D) Relative abundance of microRNA (miRNA) targets in various genotypes as measured by quantitative-real time polymerase chain reaction in 14-day-old (top) or 20-day-old (bottom) rosettes. Target genes were normalized to EIF4. Actin was used as a non-target control ( \pm standard deviation). (E) Small RNA blots of low-molecularweight RNA isolated from 14-day-old or 20-day-old rosettes probed with oligonucleotides complementary to specific miRNAs. Methionyl transfer RNA was used as a loading control.

uitin-mediated proteolysis through the $26 \mathrm{~S}$ proteasome $[22,23]$. F-box proteins bind to unique substrates and thus provide specificity to the complex. Evidence that FBW2 (SKIP18) is a component of a E3 ubiquitin ligase complex is provided by the observation that it interacts with several different components of this complex in yeast twohybrid assays [24]. We examined the effect of overexpressing FBW2 in transgenic plants, using the constitutively expressed Cauliflower Mosaic Virus 35S promoter (35S::FBW2; hereafter $F B W 2 o x$ ). This approach was suggested by the observation that over-expressing F-box pro- teins typically enhances the degradation of their protein targets [22,25-27]. The vast majority of the primary transformants we obtained in this experiment had developmental phenotypes strikingly similar to those of hypomorphic ago1 alleles, consistent with the hypothesis that FBW2 represses the activity of AGO1 (Figure 4A). In contrast, plants over-expressing three genes closely related to $F B W 2--F B L 9, F B L 20$, and SKIP1 (Additional File 1: Figure $\mathrm{S} 1 \mathrm{~B}$ )--have no noticeable phenotype (data not shown), further suggesting that FBW2 is functionally unique. 

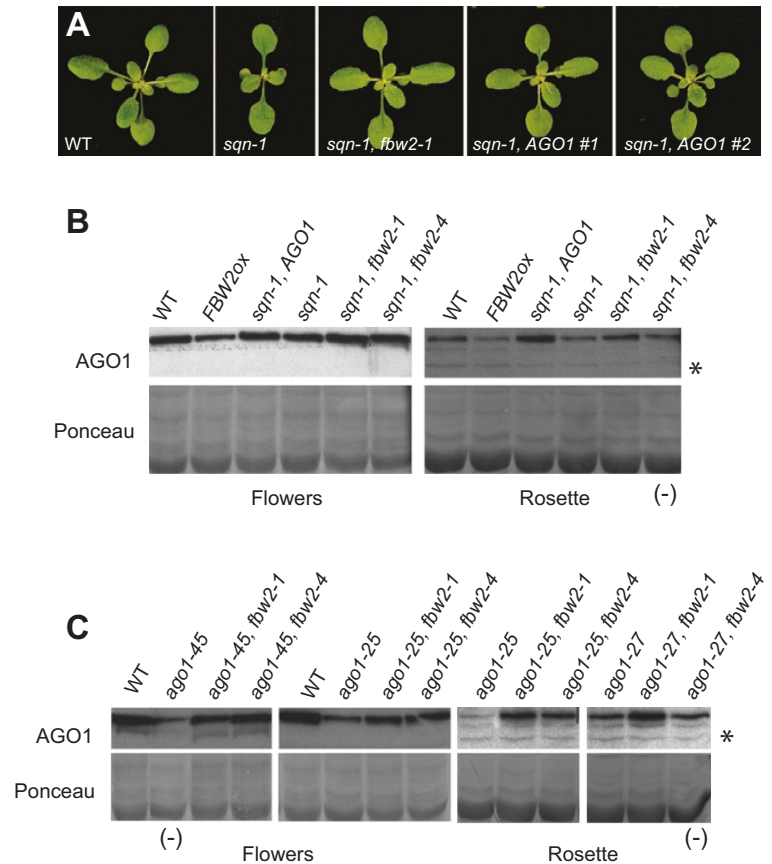

Figure 3 Mutations in FBW2 suppress sqn and ago 1 by increasing ARGONAUTE1 (AGO1). (A) Eighteen-day-old rosettes of WT, sqn-1, sqn-1 fbw2-1, and two independently isolated sqn-1 lines containing an AGO1.:FLAG-AGO1 transgene. sqn-1 AGO1.:FLAG-AGO1 transgenics resemble sqn fbw2 mutants. $(B, C)$ Western blot of protein extracts from 14-day-old rosettes or floral buds probed with an anti-AGO1 antibody. Ponceau staining and a nonspecific band $\left(^{*}\right)$ were used as a loading control. (-) indicates samples that were slightly under-loaded.

In addition to promoting miRNA-mediated silencing, AGO1 is required for at least some forms of siRNA-mediated gene silencing, including the silencing of the 35S:: GUS transgene present in the L1 line [7]. In order to determine if FBW2 affects this aspect of AGO1 function, we transformed $F B W 20 x$ into L1 plants and assayed for GUS activity in families that were homozygous for the L1 transgene and segregating $F B W 2 o x$. In contrast to L1 plants--which had low levels of GUS activity--plants containing both $L 1$ and FBW2ox had high levels of GUS activity (Figure 4B), like L1 ago1 mutants [7]. These results suggest that FBW2 affects both the miRNA- and siRNA-dependent activities of AGO1.

We tested to see if FBW2 promotes the degradation of AGO1 by examining the level of AGO1 protein in $f b w 2-1$ mutants and $F B W 2 o x$ transgenic plants. Plants overexpressing FBW2 had significantly less AGO1 than wildtype plants (Figures $2 \mathrm{~B}$ and $4 \mathrm{C}-\mathrm{D}$ ). This decrease is not explained by an effect of FBW2 on the transcription of AGO1 because AGO1 mRNA--as well as the transcripts of several other miRNA-regulated genes--is actually elevated in FBW2ox relative to wild-type plants (Figure 4E). The increase in the transcript levels of these miRNA-reg-
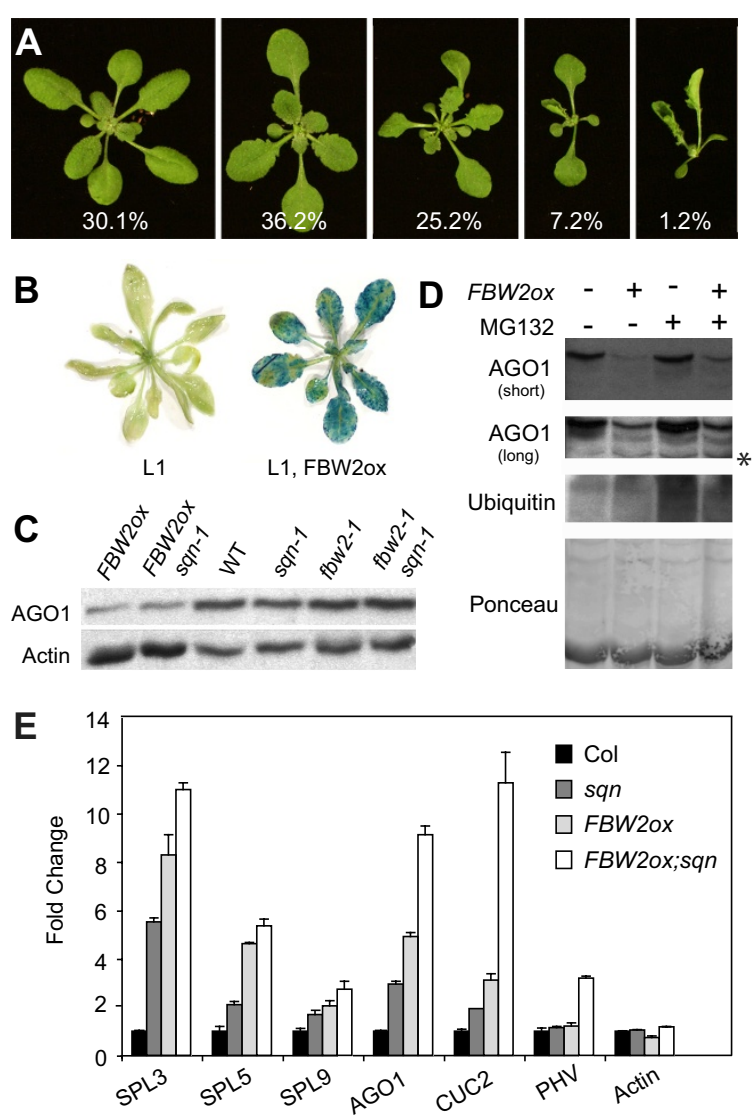

Figure 4 Over expressing FBW2 decreases ARGONAUTE1 (AGO1) protein levels. (A) Primary transformants containing a genomic 35S::FBW2 (FBW20X) transgene. The percentage of plants displaying each phenotype is indicated ( $n=345$ plants). (B) L1 plants with, and without, the FBW2Ox construct. FBW2ox restores the expression of the 355::GUS transgene present in L1. (C) Western blot of protein extracts from 14-day-old seedlings probed with an anti-AGO1 antibody. AGO1 is reduced in plants containing FBW2OX. Actin was used as a loading control. (D) The proteasome inhibitor MG132 has no effect on AGO1 protein levels. Western blots of protein isolated from plants treated as indicated were probed with anti-AGO1 antibody. The lower anti-AGO1 blot is a longer exposure. The increased abundance of ubiquitinated proteins in MG132-treated plants indicates that the treatment was effective. Ponceau staining and a nonspecific band $(*)$ were used as a loading control. (E) Abundance of miRNA targets in various genotypes as measured by quantitative-real time polymerase chain reaction. The abundance of these transcripts is significantly greater in FBW20x sqn-1 than in the parental lines, suggesting that sqn-1 and FBW2ox operate independently to reduce AGO1 activity. Results were normalized to EIF4 ( \pm standard deviation).

ulated genes is consistent with the decreased level of AGO1 protein in FBW2ox plants; AGO1 promotes miRNA-mediated gene silencing and, thus, a decrease in the abundance of this protein should lead to an increase in the level of miRNA-regulated transcripts. In contrast to its effect on the AGO1 protein, $F B W 2 o x$ had no effect on the abundance of the YFP-ZLL (AGO10) fusion protein (Additional File 2: Figure S2A)[28]. 

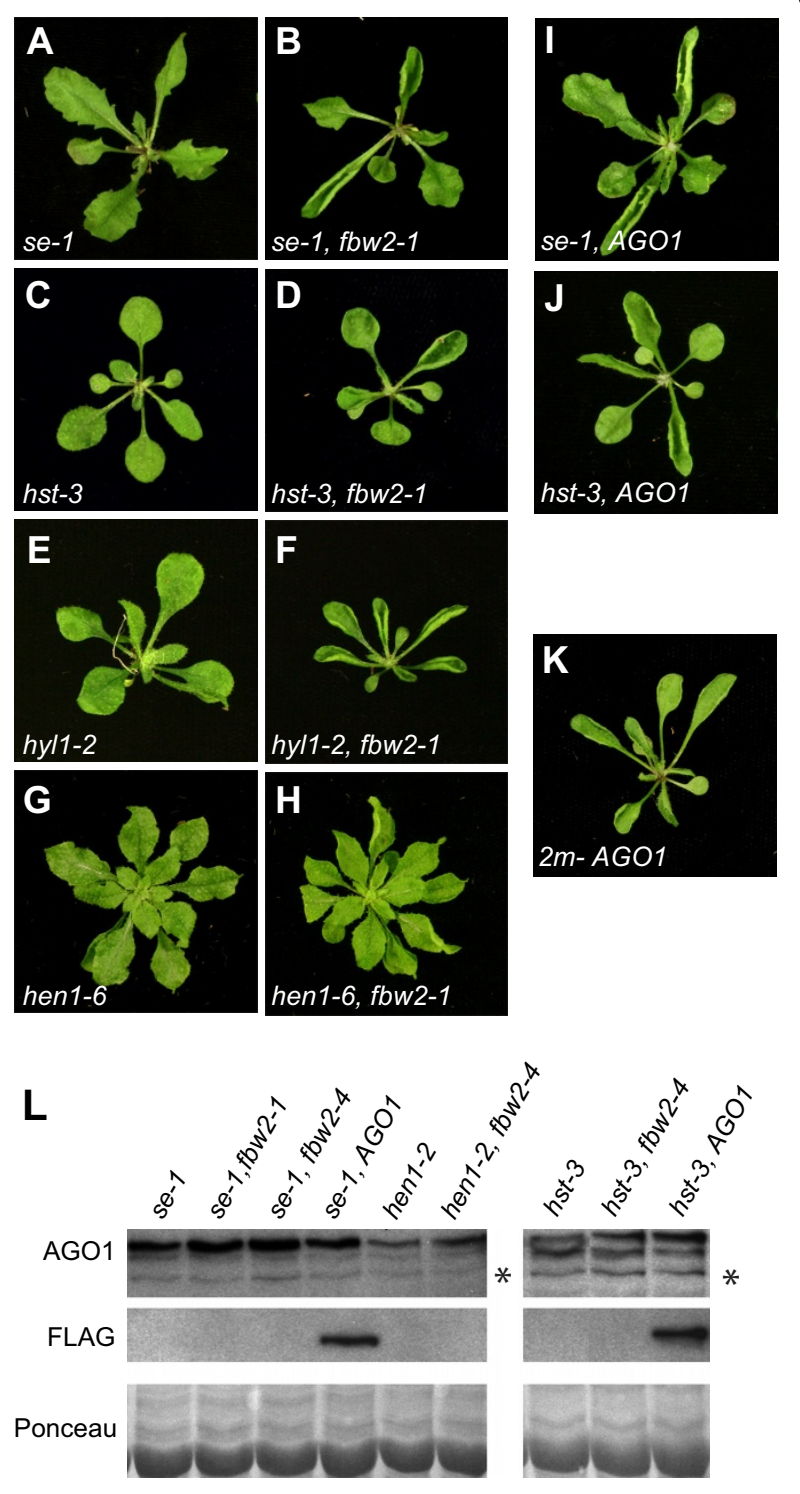

Figure 5 Mutations in FBW2 enhance the se-1, hst-3, hyl1-2 and hen 1-6 mutant phenotypes. Twenty-one-day-old plants singly and doubly mutant for fbw2-1 and hst-3 (A and B) se-1 (C and D), or hyll-2 (E and F), and 28-day-old hen 1-6 and hen 1-6 fbw2-4 plants ( $\mathrm{G}$ and $\mathrm{H}$ ). hyl13 fow $2-1$ and se- 1 fow $2-1$ double mutants have phenotypes similar to hyl1-3 and se-1 plants transformed with ARGONAUTE1.:.FLAG-AGO1 (I and J) or WT plants containing the miR168 insensitive 2m-AGO1 construct (K). (L) Western blot of protein extracts from 14 day-old seedlings probed with an anti-AGO1 antibody. Ponceau staining and a nonspecific band $\left(^{*}\right)$ were used as a loading control.

In order to determine if the FBW2-mediated decrease in AGO1 is proteasome dependent, we assayed AGO1 protein levels in the presence of the proteasome inhibitor MG132 [29]. Although treatment with MG132 produced a general decrease in protein degradation (Figure 4D), it had no effect on AGO1 protein levels in both wild-type and $F B W 2 o x$ plants (Figures $4 \mathrm{D}$ and S2B). This result sug- gests that the $35 S:: F B W 2$-mediated decrease in AGO1 is proteasome-independent and also indicates that AGO1 may be the target of an, as yet, unidentified proteasomedependent degradation pathway. Interestingly, destabilization of AGO1 by the viral F-box protein P0 is also insensitive to MG132 [19].

Although $f b w 2$ produces an observable increase in the amount of AGO1 protein in genetic backgrounds in which miRNA activity is compromised (Figures $3 \mathrm{~B}$ and $3 \mathrm{C}, 4 \mathrm{C}$ and $5 \mathrm{~L}$ ), we were unable to detect a significant increase in AGO1 protein in $f b w 2$ single mutants (Figure $4 C)$. We suspect this is because the miR168-dependent feedback mechanism that regulates AGO1 expression [13] partially corrects for slight increases in the level of this protein in $f b w 2$ mutants. Mutations that interfere with the activity of miR168 (for example, sqn, agoland hen1) disrupt this feedback mechanism, thereby making AGO1 more susceptible to other factors that regulate its expression.

\section{fbw2 is hyposensitive to abscisic acid (ABA)}

Although we were unable to observe an increase in AGO1 protein in $f b w 2$ single mutants, these mutants have a phenotype that is indicative of an increase in AGO1. Mutations that interfere with miRNA biogenesis including hst, se, hyl1, dcl1 and hen1-confer hypersensitivity to the phytohormone ABA [30,31]. These mutations inhibit both seed germination and root elongation in the presence of low levels of ABA, probably due to the misregulation of the miR159 targets MYB101 and MYB33 [32]. We reasoned that if $f b w 2$ mutations increase AGO1 levels this should result in increased miRNA activity and produce the opposite phenotype, namely, hyposensitivity to ABA. Indeed, this is what we found.

Seeds of various genotypes were grown on varying concentrations of $\mathrm{ABA}$ and scored for germination after 5 days. ago1-25 and $F B W 20 x$ were hypersensitive to ABA, with ago1-25 showing the greatest response. In the presence of ABA, both of these AGO1-deficient genotypes produced a significant decrease $(P<0.001$ at $0.75 \mu \mathrm{M}$ ABA) in seed germination relative to wild-type plants (Figure 6A); FBW2ox also displayed a slightly enhanced sensitivity to ABA in a root elongation assay (Figure 6B). Thus, AGO1 is required for a normal ABA response. In contrast, $f b w 2$ mutants displayed a significantly increased rate of germination $(P<0.01$ at $0.75 \mu \mathrm{M} A B A)$ and increased rate of root elongation in the presence ABA $(P$ $<0.03$; Figure 6A and 6B). This result provides additional support for the conclusion that FBW2 normally represses the activity of AGO1 and reveals a physiological function for FBW2.

\section{Discussion}

The results presented here demonstrate that FBW2 is a negative regulator of AGO1. We show that loss of FBW2 


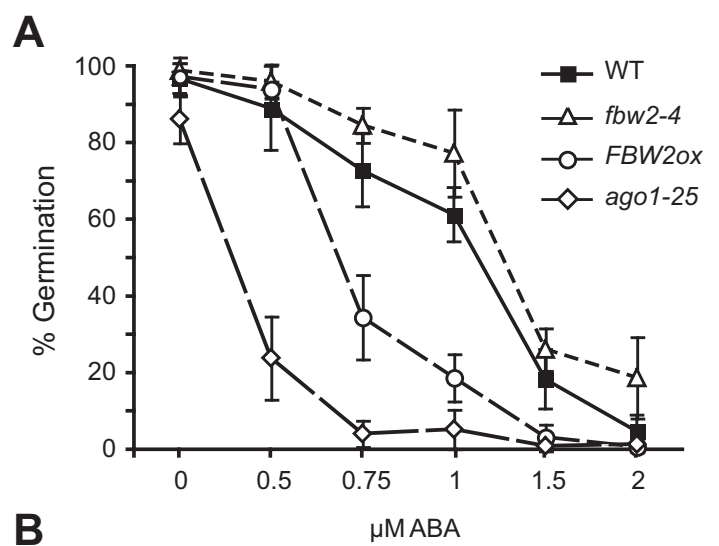

B

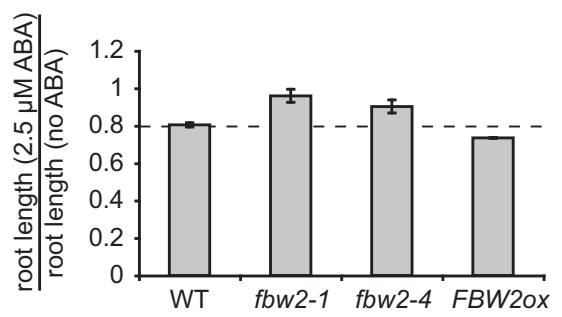

Figure 6 FBW2 is required for a proper abscisic acid (ABA) response. (A) The percent germination of WT, fbw2-4, ago 1-25 and FBW2OX seeds in the presence of increasing concentrations of ABA ( \pm standard deviation). (B) The effect of $A B A$ on root elongation, reported as the ratio of root length in the presence of $2.5 \mu \mathrm{m}$ ABA to root length in the absence of $A B A$ ( \pm standard deviation).

suppresses the phenotype of mutations that reduce the activity of AGO1 and that this effect is associated with an increase in AGO1 protein levels. Conversely, overexpressing FBW2 produces an ago1 loss-of-function phenotype and leads to a decrease in the level of AGO1. Although a loss of FBW2 does not produce a major change in the abundance of AGO1 in an otherwise wildtype background, the reduced sensitivity of $f b w 2$ mutants to ABA is consistent with a slight increase in AGO1 activity. Previous studies have demonstrated that Arabidopsis is hypersensitive to changes in AGO1 activity $[8,9,12]$ so it would not be surprising if even a minor change in the abundance of this protein is biologically significant. Over-expressing FBW2 did not produce an observable decrease in the Argonaute protein AGO10/ZWL/PNH, suggesting that FBW2 may act specifically on AGO1.

The simplest and most direct way in which FBW2 could regulate AGO1 is by binding directly to this protein, thereby directing it to a proteasome-independent degradation pathway. However, we have been unable to detect a direct interaction between FBW2 and AGO1 (data not shown). Consequently, we cannot eliminate the possibility that FBW2 acts indirectly, through its effect on a protein required for AGO1 stability. This protein cannot be
SQN because $f b w 2$ mutations rescue the phenotype of the null allele, sqn-1. Furthermore, over expressing FBW2 produces a much more severe phenotype than that of $s q n$ null alelles. Recent work suggests that the viral F-box protein, $\mathrm{P} 0$, acts by targeting an unknown component of the AGO1 RISC complex, leading to the destabilization and degradation of AGO1 [33]. It may be that FBW2 regulates this same protein or another protein within the RISC complex. Loss-of-function mutations in this hypothetical FBW2-regulated factor would be expected to have the same phenotype as FBW2ox plants, such as an ago1 lossof-function phenotype. Extensive screens for genes required for miRNA and siRNA biogenesis and activity by us and others have produced many loss-of-function alleles of AGO1 but, with the exception of sqn, have yet to reveal other ago1-like mutants. Determining the identity of this unknown protein (if it exists) may require a biochemical approach.

The activity of AGO1 in Arabidopsis is regulated by a variety of different mechanisms that act together to maintain the expression of this protein at a constant level $[9-11,13,15]$. This is critical because both an increase and a decrease in the abundance of AGO1 have significant effects on plant development. An important component of this homeostatic mechanism is the negative regulation of AGO1 by miR168 $[9,13]$. miR168 represses AGO1 in an AGO1-dependent fashion: a decrease in the activity of AGO1 leads to a decrease in miR168 activity and a subsequent increase in AGO1 expression, whereas an increase in AGO1 activity has the opposite effect. We believe this feedback loop is responsible for the observation that $f b w 2$ mutations individually have no major effect on AGO1 because these same mutations elevate AGO1 protein levels in combination with mutations that interfere with miRNA biogenesis or activity.

Such finely tuned posttranscriptional regulation of an Argonaute protein is not unique to AGO1. The stability and sub-cellular localization of the mammalian protein Ago2 are influenced by hydroxylation and phosphorylation [34,35], while the turnover of Ago2 is controlled by an E3 ubiquitin ligase [36]--a mechanism that may be quite similar to the mechanism we propose here. Furthermore, Ago2 is post-translationally controlled by a variety of environmental and developmental cues, which operate via well-defined pathways [34-36]. It would not be surprising if environmental and developmental signals also play important roles in the regulation of AGO1. The observation that $f b w 2$ has little, or no effect, on plant morphology, but decreases the sensitivity of plants to $\mathrm{ABA}$, is relevant in this case. Among other things, ABA regulates the response to water stress. Therefore, the effect of $f b w 2$ on ABA sensitivity suggests that changes in AGO1 activity may underlie the response to this and other environmental signals. The potential involvement 
of FBW2 in such regulatory pathways is an interesting subject for future studies.

\section{Conclusion}

Our results demonstrate that FBW2 is a negative regulator of AGO1 and acts by destabilizing this protein. Although we are unable to determine whether FBW2 destabilizes AGO1 directly or via an effect on an as yet unknown protein, these results add yet another layer of control to the already complex mechanism responsible for AGO1 homeostasis. Loss of FBW2 affects the sensitivity of plants to the growth regulator ABA, suggesting a possible role for FBW2 in hormone response pathways.

\section{Methods}

\section{Genetic stocks and growth conditions}

Unless otherwise noted, all mutations described in this paper are in the Columbia background. $f b w 2-1, f b w 2-2$ and $f b w 2-3$ were identified in M2 families of EMS-mutagenized sqn-1 plants. Primers for genotyping can be found in Additional File 3: Table S1. ago1-45 and ago1-46 have been previously described [12]. ago1-36 plants containing pGreen0229 AGO1::FLAG-AGO1 were provided by D Baulcombe (University of Cambridge, Cambridge, UK) [21]. ago1-25, ago1-27, 2m-AGO1 lines and L1 lines were provided by $\mathrm{H}$ Vaucheret (Institut National de la Recherche Agronomique, Versailles, France) [7,8]. Plants containing YFP-AGO10 ( $p Z L L:: Y F P-Z L L)$ in the Ler background were obtained from T Laux [28]. $f b w 2-4$ (SALK_144548), fbw2-5 (SALK_071588), hen1-2 (SALK_090960), hyl1-2 (SALK_064863) and se-1 were obtained from the Arabidopsis Biological Resource Center http://abrc.osu.edu/[18]. Unless otherwise noted, plants were grown on Farfard soil at $23^{\circ} \mathrm{C}$ under $16 \mathrm{~h}$ fluorescent illumination. Wild-type or $f b w 2$ plants segregating ago1-36 were grown on Murashigi and Skoog (MS) plates $(0.5 \% \mathrm{MS}, 1 \%$ sucrose, $0.8 \%$ agar $\mathrm{pH}$ 5.7). GUS assays were performed as previously described [37].

\section{RT-PCR}

Total RNA was isolated using TRIzol (Invitrogen, CA, USA) from total above-ground tissue of 14-day-old seedlings. RNA was immobilized on RNeasy Plant Columns (Qiagen, CA, USA) and treated with DNAse (Qiagen). cDNA was amplified from 1-4 ug of RNA using Superscript II (Invitrogen). Quantitative RT PCR was performed using Sybr Green PCR Master Mix (Applied Biosystems, CA, USA) on a StepOnePlus ${ }^{\text {tw }}$ RT-PCR System (Applied Biosystems). FBW2 RT Primers are described in Additional File 3: Table S1. Primers for RTPCR of miRNA targets were previously described [12]. Transcript levels were normalized against EIF4 levels in all experiments.

\section{RNA blots}

RNA blots were generated and analysed as previously described [12,38]. Briefly, total RNA was isolated using TRIzol (Invitrogen) from total above-ground tissue of 14day-old seedlings. High molecular weight RNA was removed by precipitating with PEG-8000 (5\%) and $\mathrm{NaCl}$ $(500 \mathrm{mM})$. Low molecular weight (LMW) RNA was subsequently precipitated with $300 \mathrm{mM} \mathrm{NaOAc}$ and 2 vol $100 \% \mathrm{EtOH}$ and washed with 70\% EtOH. LMW RNA was separated on an $8 \mathrm{M}$ urea/15\% denaturing polyacrylamide gel and was transferred to a Hybond $\mathrm{N}$ membrane (Amersham Pharmacia, NJ, USA). Oligonucleotide probes were labelled using T4 polynucleotide kinase (New England Biolabs, MA, USA) with [ $\gamma-32 \mathrm{P}]-\mathrm{ATP}$ at $40^{\circ} \mathrm{C}$ in ULTRAhyb-oligo hybridization buffer (Ambion, CA, USA). Membranes were hybridized with oligonucleotide probes complementary to specific miRNA targets.

\section{Western blot and immunoprecpitation experiments}

Fourteen-day-old, 20-day-old seedlings, or floral buds were ground using liquid nitrogen and resuspended in 1:3 w/v extraction buffer [ $20 \mathrm{mM}$ Tris pH 7.5, $300 \mathrm{mM} \mathrm{NaCl}$, $5 \mathrm{mM} \mathrm{MgCl}_{2}, 1 \times$ Protease Inhibitor Cocktail (Sigma, MO, USA) $1 \mathrm{mM}$ PMSF, $1 \mathrm{mM}$ DTT]. Equal amounts of soluble protein were separated on an 8.5\% SDS-PAGE gel, transferred to a nitrocellulose membrane and membranes were blocked [tris buffered saline with tween (TBS-T) with 5\% milk]. Anti-AGO1 (1:500; antibody provided by Y Qi and Xioafeng Cao, National Institute of Biological Sciences, Beijing, China), anti-FLAG monoclonal (1:2000) (Sigma - F1804), anti-FLAG HRP conjugated (1:2000; Sigma - A8592), anti-GFP (Invitrogen - A-6455), anti-cMyc rabbit (1:2000; Sigma - C3956), were incubated overnight in TBS-T $+5 \%$ milk at $4^{\circ}$. Anti-actin $(1: 10,000)$ (Sigma - A0480) was incubated at room temperature for 2 h.

\section{Constructs}

The FBW2 coding or FBW2 genomic region was amplified by PCR using the $F B W 2$ primers (Additional File 3: Table S1). PCR products were TOPO cloned into pENTR-D TOPO (Invitrogen). The $F B W 2$ coding and the 4701-base pair genomic $F B W 2$ genomic region were recombined into pEG100 (35S) and pEG302 (FLAG), respectively [39]. For $A G O 1:: F L A G-A G O 1$ constructs, genomic AGO1::FLAG-AGO was amplified from the pGreen0229 FLAG-AGO1 construct using AGO1 F and $\mathrm{R}$ primers (Additional File 3: Table S1) [21]. PCR products were cloned into pENTR-D TOPO vector and recombined into the Cambia 3301 vector containing a GATEWAY cassette. All constructs were transformed using the Floral Dip technique [40]. 


\section{ABA experiments}

Sterilized seeds were plated on $1 \%$ sucrose LS plates containing $0,0.5,0.75,1.0,1.5$ or $2.0 \mu \mathrm{M}$ ABA, imbibed for 3 days in the dark at $4^{\circ} \mathrm{C}$ and grown for 5 days under long day conditions ( $16 \mathrm{~h}$ light, $8 \mathrm{~h}$ dark). Plants were scored for greening of the cotyledons. Experiments were repeated at least three times. In order to determine the effect of ABA on root growth, sterilized seeds were plated vertically on $1 \%$ sucrose LS plates, imbibed for 3 days, and grown for 5 days under long day conditions $(16 \mathrm{~h}$ light, $8 \mathrm{~h}$ dark). Seedlings of equal size were then transplanted to vertical plates containing either $0 \mu \mathrm{M}$ or 2.5 $\mu \mathrm{M} A B A$, and grown for 5 days or more under long day conditions (16 h light, $8 \mathrm{~h}$ dark). Primary root length was measured for each plant and root inhibition was determined as the average root length of plants grown at 2.5 $\mu \mathrm{M}$ ABA relative to the average root length of plants gown at $0 \mu \mathrm{M} \mathrm{ABA}$.

\section{Additional material}

Additional file 1 Figure S1. (A) Gene structure of FBW2 showing the location of primers used in real time polymerase chain reaction and the abundance of FBW2 messenger RNA in sqn-1 and mutants doubly mutant for sqn-1 and different alleles of fbw2. All target genes were normalized to EIF4. (B) Alignments of the Arabidopsis F-box genes most closely related to At4g08980 (FBW2): At4g05497 (FBW9), At4g05460 (FBL20), At5g57900 (SKIP1). (C) Alignments of the amino acid sequences of FBW2 and its predicted orthologs in other flowering plants. All alignments were made using the following assembled contigs from PlantGDB unless otherwise noted: Citrus sinensis (PUT-157a-Citrus_sinensis-6728477), Gossypium raimondii (PUT-157a-Gossypium_raimondii-11427), Glycine max (PUT-161aGlycine_max-76896), Oryza sativa Japonica (Genbank AK070008), Asparagus officinalis (Genbank CV288647), Vitis vinifera (PUT-157a-Vitis_vinifera-16230), Nicotiana tabacum (PUT-163a-Nicotiana_tabacum-55992858), Lactuca sativa (PUT-157a-Lactuca_sativa-30499), Pinus taeda (PUT-157aPinus_taeda-79282550)

Additional file 2 Figure S2. (A) Western blot comparing levels of ARGONAUTE1 (AGO1) and yellow fluorescent protein-AGO10 in plants with and without FBW2Ox constructs. Anti-AGO1 and anti-GFP antibodies were used to probe each blot. Ponceau staining was used as a loading control. (B) Levels of AGO1-FLAG do not decrease in plants treated with the protease inhibitor MG132. Blots were probed with anti-FLAG monoclonal antibody or anti-ubiquitin antibody. The anti-ubiquitin antibody demonstrates an overall increase in ubiquitination in MG132 treated plants, as expected from a decrease in proteasome activity.

Additional file $\mathbf{3}$ Table S1. Polymerase chain reaction primers used in this study.

\section{Abbreviations}

ABA: abscisic acid; AGO1: ARGONAUTE1; EMS: ethyl methanesulfonate; mRNA: messenger RNA; miRNA: microRNA; RISC: RNA-induced silencing complex; RT$P C R$ : real time polymerase chain reaction; siRNA: small interfering RNA; SQN: SQUINT; TBS-T: tris buffered saline tween.

\section{Competing interests}

The authors declare that they have no competing interests.

\section{Authors' contributions}

KWE performed most of the work described in this paper and co-wrote the manuscript with RSP. MRS performed the sqn suppressor screen. MRS and KWE mapped FBW2; RW performed the ABA experiments under the guidance of BDG.

\section{Acknowledgements}

We are grateful to Matthew Willmann and Stewart Gillmor for their comments on this manuscript. This work is supported by NIH Grant RO1GM051893 to RSP. KWE is supported by an NIH NRSA postdoctoral fellowship (F32GM084591) from the NIGMS. The content is solely the responsibility of the authors and does not necessarily represent the official views of the National Institute of General Medical Sciences or the National Institutes of Health.

\section{Author Details}

Department of Biology, University of Pennsylvania, Philadelphia PA 19104, USA

Received: 23 March 2010 Accepted: 12 July 2010

Published: 12 July 2010

\section{References}

1. Hutvagner G, Simard MJ: Argonaute proteins: key players in RNA silencing. Nat Rev Mol Cell Biol 2008, 9(1):22-32.

2. Vaucheret H: Plant ARGONAUTES. Trends Plant Sci 2008, 13(7):350-358.

3. Voinnet O: Origin, biogenesis and activity of plant microRNAs. Cell 2009, 136(4):669-687

4. Carthew RW, Sontheimer EJ: Origins and mechanisms of miRNAs and siRNAs. Cell 2009, 136(4):642-655.

5. Bohmert K, Camus I, Bellini C, Bouchez D, Caboche M, Benning C: AGO1 defines a novel locus of Arabidopsis controlling leaf development. EMBO J 1998, 17(1):170-180.

6. Kidner CA, Martienssen RA: Spatially restricted microRNA directs leaf polarity through ARGONAUTE1. Nature 2004, 428(6978):81-84

7. Fagard M, Boutet S, Morel JB, Bellini C, Vaucheret H: AGO1, QDE-2, and $\mathrm{RDE}-1$ are related proteins required for post-transcriptional gene silencing in plants, quelling in fungi, and RNA interference in animals. Proc Natl Acad Sci USA 2000, 97(21):11650-11654.

8. Morel JB, Godon C, Mourrain P, Beclin C, Boutet S, Feuerbach F, Proux F, Vaucheret $\mathrm{H}$ : Fertile hypomorphic ARGONAUTE (ago1) mutants impaired in post-transcriptional gene silencing and virus resistance. Plant Cell 2002, 14(3):629-639.

9. Vaucheret $H$, Vazquez $F$, Crete $P$, Bartel DP: The action of ARGONAUTE1 in the miRNA pathway and its regulation by the miRNA pathway are crucial for plant development. Genes Dev 2004, 18(10):1187-1197.

10. Mi S, Cai T, Hu Y, Chen Y, Hodges E, Ni F, Wu L, Li S, Zhou H, Long C, et al.: Sorting of small RNAs into Arabidopsis argonaute complexes is directed by the $5^{\prime}$ terminal nucleotide. Cell 2008, 133(1):116-127.

11. Takeda A, Iwasaki S, Watanabe T, Utsumi M, Watanabe Y: The mechanism selecting the guide strand from small RNA duplexes is different among argonaute proteins. Plant Cell Physiol 2008, 49(4):493-500.

12. Smith MR, Willmann MR, Wu G, Berardini TZ, Moller B, Weijers D, Poethig RS: Cyclophilin 40 is required for microRNA activity in Arabidopsis. Proc Natl Acad Sci USA 2009, 106(13):5424-5429.

13. Vaucheret $H$, Mallory AC, Bartel DP: AGO1 homeostasis entails coexpression of MIR168 and AGO1 and preferential stabilization of miR168 by AGO1. Mol Cell 2006, 22(1):129-136.

14. Mallory AC, Vaucheret $H$ : ARGONAUTE 1 homeostasis invokes the coordinate action of the microRNA and siRNA pathways. EMBO Rep 2009, 10(5):521-526.

15. Mallory AC, Hinze A, Tucker MR, Bouche N, Gasciolli V, Elmayan T, Lauressergues D, Jauvion V, Vaucheret H, Laux T: Redundant and specific roles of the ARGONAUTE proteins AGO1 and ZLL in development and small RNA-directed gene silencing. PLoS Genet 2009, 5(9):e1000646.

16. Berardini TZ, Bollman K, Sun H, Poethig RS: Regulation of vegetative phase change in Arabidopsis thaliana by Cyclophilin 40. Science 2001, 291(5512):2405-2407

17. Xiao W, Jang J: F-box proteins in Arabidopsis. Trends Plant Sci 2000 5(11):454-457

18. Alonso JM, Stepanova AN, Leisse TJ, Kim CJ, Chen H, Shinn P, Stevenson DK, Zimmerman J, Barajas P, Cheuk R, et al.: Genome-wide insertional mutagenesis of Arabidopsis thaliana. Science 2003, 301(5633):653-657.

19. Baumberger N, Tsai CH, Lie M, Havecker E, Baulcombe DC: The Polerovirus silencing suppressor P0 targets ARGONAUTE proteins for degradation. Curr Biol 2007, 17(18):1609-1614

20. Bortolamiol D, Pazhouhandeh M, Marrocco K, Genschik P, Ziegler-Graff V: The Polerovirus $\mathrm{F}$ box protein P0 targets ARGONAUTE1 to suppress RNA silencing. Curr Biol 2007, 17(18):1615-1621. 
21. Baumberger N, Baulcombe DC: Arabidopsis ARGONAUTE1 is an RNA Slicer that selectively recruits microRNAs and short interfering RNAs. Proc Natl Acad Sci USA 2005, 102(33):1 1928-11933.

22. Lechner E, Achard P, Vansiri A, Potuschak T, Genschik P: F-box proteins everywhere. Curr Opin Plant Biol 2006, 9(6):631-638.

23. Ho MS, Ou C, Chan YR, Chien CT, Pi H: The utility F-box for protein destruction. Cell Mol Life Sci 2008, 65(13):1977-2000

24. Risseeuw EP, Daskalchuk TE, Banks TW, Liu E, Cotelesage J, Hellmann H, Estelle M, Somers DE, Crosby WL: Protein interaction analysis of SCF ubiquitin E3 ligase subunits from Arabidopsis. Plant $J$ 2003, 34(6):753-767.

25. Coates JC, Laplaze L, Haseloff J: Armadillo-related proteins promote lateral root development in Arabidopsis. Proc Natl Acad Sci USA 2006, 103(5):1621-1626.

26. Dong L, Wang L, Zhang Y, Deng $X$, Xue Y: An auxin-inducible F-box protein CEGENDUO negatively regulates auxin-mediated lateral root formation in Arabidopsis. Plant Mol Biol 2006, 60(4):599-615.

27. Nelson DC, Lasswell J, Rogg LE, Cohen MA, Bartel B: FKF1, a clockcontrolled gene that regulates the transition to flowering in Arabidopsis. Cell 2000, 101(3):331-340.

28. Tucker MR, Hinze A, Tucker EJ, Takada S, Jurgens G, Laux T: Vascular signalling mediated by ZWILLE potentiates WUSCHEL function during shoot meristem stem cell development in the Arabidopsis embryo. Development 2008, 135(17):2839-2843.

29. Myung J, Kim KB, Crews CM: The ubiquitin-proteasome pathway and proteasome inhibitors. Med Res Rev 2001, 21(4):245-273.

30. Lu C, Fedoroff N: A mutation in the Arabidopsis HYL1 gene encoding a dsRNA binding protein affects responses to abscisic acid, auxin, and cytokinin. Plant Cell 2000, 12(12):2351-2366.

31. Zhang JF, Yuan LJ, Shao Y, Du W, Yan DW, Lu YT: The disturbance of small RNA pathways enhanced abscisic acid response and multiple stress responses in Arabidopsis. Plant Cell Environ 2008, 31(4):562-574.

32. Reyes $\mathrm{JL}$, Chua NH: ABA induction of miR159 controls transcript levels of two MYB factors during Arabidopsis seed germination. Plant $J 2007$, 49(4):592-606.

33. Csorba T, Lozsa R, Hutvagner G, Burgyan J: Polerovirus protein PO prevents the assembly of small RNA containing RISC complexes and leads to degradation of ARGONAUTE1. Plant J 2010, 62(3):463-472.

34. Qi HH, Ongusaha PP, Myllyharju J, Cheng D, Pakkanen O, Shi Y, Lee SW, Peng J: Prolyl 4-hydroxylation regulates Argonaute 2 stability. Nature 2008, 455(7211):421-424.

35. Zeng Y, Sankala H, Zhang X, Graves PR: Phosphorylation of Argonaute 2 at serine-387 facilitates its localization to processing bodies. Biochem J 2008, 413(3):429-436.

36. Rybak A, Fuchs H, Hadian K, Smirnova L, Wulczyn EA, Michel G, Nitsch R, Krappmann D, Wulczyn FG: The let-7 target gene mouse lin- 41 is a stem cell specific E3 ubiquitin ligase for the miRNA pathway protein Ago2. Nat Cell Biol 2009, 11(12):1411-1420.

37. Donnelly PM, Bonetta D, Tsukaya H, Dengler RE, Dengler NG: Cell cycling and cell enlargement in developing leaves of Arabidopsis. Dev Biol 1999, 215(2):407-419.

38. Park MY, Wu G, Gonzalez-Sulser A, Vaucheret H, Poethig RS: Nuclear processing and export of microRNAs in Arabidopsis. Proc Natl Acad Sci USA 2005, 102(10):3691-3696.

39. Earley KW, Haag JR, Pontes O, Opper K, Juehne T, Song K, Pikaard CS: Gateway-compatible vectors for plant functional genomics and proteomics. Plant J 2006, 45(4):616-629.

40. Clough SJ, Bent AF: Floral dip: a simplified method for Agrobacteriummediated transformation of Arabidopsis thaliana. Plant J 1998, 16(6):735-743.

doi: $10.1186 / 1758-907 X-1-15$

Cite this article as: Earley et al., An endogenous F-box protein regulates ARGONAUTE1 in Arabidopsis thaliana Silence 2010, 1:15

\section{Submit your next manuscript to BioMed Central} and take full advantage of:

- Convenient online submission

- Thorough peer review

- No space constraints or color figure charges

- Immediate publication on acceptance

- Inclusion in PubMed, CAS, Scopus and Google Scholar

- Research which is freely available for redistribution

Submit your manuscript at www.biomedcentral.com/submit
C BioMed Central 